CARTA AL EDITOR

\title{
Manía psicótica inducida por olanzapina en el trastorno esquizoafectivo bipolar
}

\author{
F. Benazzi \\ Departamento de Psiquiatría, Servicio National de Salud, Hospital Público Morgagni, Forli, Italia
}

Se ha comunicado en ocho pacientes con esquizofrenia $[1,4,6,7,9,10]$ y en dos pacientes con trastorno esquizoafectivo no bipolar $[2,4]$ que el antipsicótico atípico olanzapina induce agitación maníaca. No se encontraron informes de manía inducida por olanzapina en el trastorno bipolar en Medline o en Focus on Psychopharmacology (ISI). Se presenta a una paciente con trastorno esquizoafectivo bipolar que tuvo un episodio de manía psicótica inducido por olanzapina.

Una mujer de 55 años con una historia de 20 años de trastorno esquizoafectivo bipolar del DSM-IV, con síntomas principalmente negativos no graves durante meses, había sido tratada con $6 \mathrm{mg} / \mathrm{d}$ de haloperidol, $100 \mathrm{mg} / \mathrm{d}$ de levopromacina y $30 \mathrm{mg} / \mathrm{d}$ de flurazepam durante seis meses. Para mejorar el cuadro clínico, se la cambió sin reducción progresiva de las dosis a 20 $\mathrm{mg} / \mathrm{d}$ de olanzapina y $2 \mathrm{mg} / \mathrm{d}$ de clonazepam. Una semana después tenía insomnio grave, agitación psicomotriz, irritabilidad e hiperactividad. Unos días después fue hospitalizada por agresividad grave con agresiones al personal, ideas delirantes megalomaníacas y habla a presión. Se interrumpió la olanzapina y su estado remitió en unos días con $600 \mathrm{mg} / \mathrm{d}$ de valproato, $6 \mathrm{mg} / \mathrm{d}$ de haloperidol y $10 \mathrm{mg} / \mathrm{d}$ de lorazepam.

Aunque no se puede excluir un cambio maníaco espontáneo, la estrecha asociación temporal entre el curso de la manía psicótica y el tratamiento con olanzapina, junto con la breve duración del episodio (se espera que una recaída espontánea de la manía psicótica dure más y no tenga un comienzo y una terminación abrupta), hacen pensar en una relación causal. Es poco probable que la interrupción repentina del halo- peridol haya inducido una manía de rebote, porque este medicamento tiene una semivida cerebral muy larga [3]. Los fármacos sedantes levopromacina (un neuroléptico) y flurazepam (una benzodiacepina) se sustituyeron por la benzodiacepina sedante clonazepam, impidiendo un síndrome de supresión de benzodiacepinas (que, si es grave, podría tener algunos síntomas maníacos). Se ha informado de la inducción de manía por el antipsicótico atípico risperidona [5]. Tanto la risperidona como la olanzapina tienen un antagonismo mayor con la serotonina (5-HT2A) que con la dopamina (D2), pero las afinidades relativas para éstos y otros receptores son diferentes [11]. La risperidona puede inducir manía por bloqueo de 5-HT2 [8]. La olanzapina podría haber inducido manía psicótica en este caso en una manera similar.

\section{BIBLIOGRAFÍA}

1 Jeshi A. Paranola and agitation with olanzapine treatment [letter]. Can J Psychiatry 1998; 43: 195.

2 Benazzi F, Rossi E. Mania induced by olanzapine [letter]. Hum Psychopharmacol Clin Exp 1998; 13: 585-6.

3 Cohen BM, Babb S, Campbell A, Baldessarini RJ. Persistence of haloperidol in the brain [letter]. Arch Gen Psychiatry 1988; 45: 879-80.

4 John V, Rapp M, Pies R. Aggression, agitation, and mania with olanzapine [letter]. Can J Psychiatry 1998; 43: 1054.

5 Lane HY, Lin YC, Chang VM. Mania induced by risperidone: dose related? [letter]. J Clin Psychiatry 1998; 59: 85-6.

6 Lindenmayer JP, Klebanov R. Olanzapine-induced, manic-like syndrome [letter]. J Clin Psychiatry 1998; 59: $318-9$. 
7 London JA. Mania associated with olanzapine [letter]. J Am Acad Child Adolesc Psychiatry 1998; 37: 135-6.

8 McElroy SL, Keck PE, Strakowski SM. Mania, psychosis, and antipsychotics. J Clin Psychiatry 1996; 57 Suppl 3: 14-26.

9 Pozo P, Alcantara AG. Mania-Ilke syndrome in a patient with chronic schizophrenia during olanzapine treatment [letter]. J Psychiatry Neurosci 1998; 23: 309-10.

10 Reeves RR, McBride WA, Brannon GE. Olanzapine-induced mania. J Am Osteopath Assoc 1998; 98: 549-50.

11 Tran PV, Hamilton SH, Kuntz AJ, Potvin JH, Andersen SW, Beasley C, et al. Double-blind comparison of olanzapine versus risperidone in the treatment of schizophrenia and other psychotic disorders. J Clin Psychopharmacol 1997; 17: $407-18$.

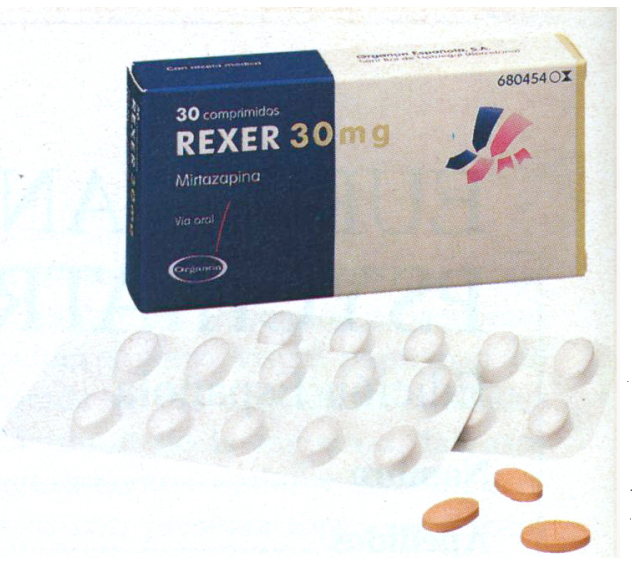

REXER: Comprimidos de $30 \mathrm{mg}$ de mirtazapina. Indicaciones: Episodio de depresión mayor. Posologia y forma de administración: Via oral, tragar con o sin liquido, sin masticar. Adultos: Inicio con $15 \mathrm{mg} /$ dia, aumentando hasta
obtener una respuesta óptima. La dosis eficaz suele estar entre 15 y $45 \mathrm{mg} / \mathrm{dia}$. Ancianos: La dosis recomendada es la misma que para los adultos, pero el aumento de dosis debe realizarse bajo supervisión. Niños: No se ha aclaramiento de mistazapina puede disminuir en pacientes con insuficiencia hepática o renal Mirtazapina tiene una semivida de 20-40 horas, por lo que puede administrarse como dosis única, preferiblemente antes de acostarse. También puede administrarse en dos subdosis divididas a partes iguales durante la jornada (mañana y noche). Es recomendable continuar el tratamiento durante $4-6$ meses más desde la ausencia de sintomas y puede finalizarse durante 4-6 meses más desde la ausencia de sintomas y puede finalizarse gradualmente. El tratamiento con dosis adecuada deberá proporcionar respuesta positiva en 2-4 semanas. Si la respuesta es insuficiente, la dosis puede 4 semanas deberá is mandonarse el tratamiento Contraindicaciones: 4 semanas, deberá abandonarse el tratamiento. Contraindicaciones: Hipersensibilidad a la mirtazapina. Advertencias y precauciones: Durante el tratamiento con muchos antidepresivos se ha descrito depresión de la medula ósea, que suele evidenciarse como granulocitopenia o agranulocitosis, casi siempre aparece después de 4-6 semanas, siendo en general reversible una vez suspendido el tratamiento. Tambien se ha informado de agranulocitosis reversible como acontecimiento adverso raro en estudios clínicos con Rexer". Deberá vigilarse la aparición de fiebre, dolor de garganta, estomatitis u otros signos de infección; si se presentan tales sintomas debera suspenderse el tratamiento y realizarse un hemograma. En los siguientes casos es necesario establecer cuidadosamente la pauta posológica y realizar un seguimiento regular: epilepsia y sindrome afectivo orgánico (aunque raramente se producen ataques en pacientes tratados con Rexer"), insuficiencia hepática o renal, ennermedades cardlacas como alteraciones de la conducción, angina de pecho e infarto de miocardio reciente (situaciones en las que deberán tomarse las precauciones habituales y administrar con precaución los medicamentos concomitantes) e hipotension. Al igual que con otros antidepresivos deben tomarse precauciones en pacientes que se encuentren en las siguientes situaciones: alteraciones de la micción por hipertrofia prostática y glaucoma de ángulo agudo con presión intraocular elevada (aunque en ambos casos no es de esperar que se produzcan problemas debido a que Rexer posee una actividad anticolinergica muy debil, y diabetes melitus. Suspender e tratamiento si se presenta ictericia. Ademas, al igual que con otros antidepresivos, debe tenerse en cuenta que puede darse un empeoramiento de los sintor esquizoffenia u otras alteraciones psicoticas, pueden intensifarse los pensamientos paranoides, puede revertirse a la fase maniaca si se trata la fase

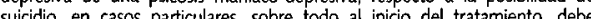
suicidio, en casos particulares, sobre todo al inicio del tratamiento, debe proporcionarse al paciente un número limitado de comprimidos de Rexer. Aunque los antidepresivos no producen adicion, la suspension brusca de trátamiento despues de la administración a largo plazo puede causar náuseas, dolor de cabeza y malestar. A veces los pacientes ancianos son más sensibles a los efectos de los antidepresivos, sobre lodo en cuanto a los efectos adversos, durante la investigación clinica con Rexer" no se han recogido diferencias entre los grupos de edad en la aparión de efectos adnos, sh embango la experiencia hasta el momento es limitada. Interactiones con otros medicamentos y otras fonas de interaccion. Mirtazapina puede potenciar la acción depresva del alcohol sobre el sistema nenioso central, por tanto los pacientes deben evitar el alcohol durante el tratamiento con Rexpo Rexen debe administrarse simultáneamente con inhibidores de la MAO o en las dos semanas posterions a la finalizacion del tratamiento con estos agentes. Mirtazapina puede potenciar los efectos sedantes de las benzodiacepinas. Embarazo y lactancia. Aunque los estudios en animales no han mostrado ningun efecto teratogenico con trascendencia toxicologica, no se ha establecido la seguridad de Rexer en el embarazo humano. Rexer se utilizara en el embarazo sólo si la necesidad es clara. Aunque los experimentos en animales muestran que mirtazapina se excreta en cantidades muy pequeñas por la leche, el uso de Rexer" en mujeres lactantes no es aconsejable por no existir datos sobre la excreción por la leche humana. Efectos sobre la capacidad para conducir vehiculos y utilizar maquinaria: Rexer puede disminuir la concentración y la alerta. Los pacientes en tratamiento con antidepresivos deben evitar realizar actjidades potencialmente peligrosas que requieran un estado de alerta y concentración, como conducir un vehiculo a motor o manejar maquinaria. Reacciones adversas: Las reacciones adversas más frecuentes durante el tratamiento con Rexer son: aumento de apetito y aumento de peso, somnolencia/sedación, generalmente durante las primeras semanas de tratamiento (Nota: En general, a reducción de dosis no producirá menor sedación y además puede comprometer la eficacia antidepresiva). En casos raros puede presentarse hipotensión (ortostática), mania, convulsiones (ataques), temblores, miodonía, edema, depresión aguda de la médula ósea (eosinofilia, granulocitopenia, agranulocitosis, anemia aplásica y trombocitopenia) (ver "Advertencias y precauciones"), aumento en las transaminasas séricas y/o exantema. Sobredosificación: No se ha establecido la seguridad clínica de Rexers por intoxicación. Los estudios de toxicidad no mostraron efectos cardiotóxicos relevantes en intoxicación con Rexer", y en los ensayos clinicos tampoco se observaron efectos de importancia dinica por sobredosificación, aparte de sedación excesiva. La sobredosificación deberá tratarse mediante lavado gástrico más una terapia sintomática apropiada y apoyo de las funciones vitales. Incompatibilidades: Ninguna. Presentaciones: Los comprimidos se presentan en blisters de seguridad a prueba de niños, con película opaca de cloruro de polivinilo de color blanco y lámina de aluminio, en envases de 30 comprimidos rojo pardo de $30 \mathrm{mg}$ de mirtazapina. PVPIVA(MR): 7.660,- Pts. Instrucciones de uso/manipulación: Ninguna en especial, Condiciones de prescripción y dispensación. Prestación farmacéutica del SNS Con receta médica. Incluido en la prestación. Aportación reducida. RUM1060.060.101/6-PU. ORGANON ESPANNOLA, 5.A. Ctra. Hospitalet, 147-149, 